\title{
ON THE GENERALIZED DETERIORATED POSITIVE SEMI-DEFINITE AND SKEW-HERMITIAN SPLITTING PRECONDITIONER*
}

\author{
Davod Hezari and Vahid Edalatpour \\ Faculty of Mathematical Sciences, University of Guilan, Rasht, Iran \\ Email:d.hezari@gmail.com,vedalat.math@gmail.com \\ Hadi Feyzollahzadeh \\ Department of Mathematical and Computer Science, Technical Faculty, \\ University of Bonab, Bonab, Iran \\ Email: hadi.feyz@yahoo.com \\ Davod Khojasteh Salkuyeh \\ Faculty of Mathematical Sciences, University of Guilan, Rasht, Iran \\ Email: khojasteh@guilan.ac.ir
}

\begin{abstract}
For nonsymmetric saddle point problems, Huang et al. in [Numer. Algor. 75 (2017), pp. 1161-1191] established a generalized variant of the deteriorated positive semi-definite and skew-Hermitian splitting (GVDPSS) preconditioner to expedite the convergence speed of the Krylov subspace iteration methods like the GMRES method. In this paper, some new convergence properties as well as some new numerical results are presented to validate the theoretical results.
\end{abstract}

Mathematics subject classification: $65 \mathrm{~F} 10,65 \mathrm{~N} 22$.

Key words: Saddle point problem, Preconditioner, Nonsymmetric, Symmetric, Positive definite, Krylov subspace method.

\section{Introduction}

Consider the solution of large sparse saddle point problems of the form

$$
\mathcal{A} u \equiv\left(\begin{array}{cc}
A & B^{T} \\
-B & 0
\end{array}\right)\left(\begin{array}{l}
x \\
y
\end{array}\right)=\left(\begin{array}{c}
f \\
-g
\end{array}\right) \equiv b,
$$

where $A \in \mathbb{R}^{n \times n}$, the matrix $B \in \mathbb{R}^{m \times n}$ is of full row rank with $m \leq n, B^{T}$ denotes the transpose of the matrix $B$. Moreover, $x, f \in \mathbb{R}^{n}$ and $y, g \in \mathbb{R}^{m}$. We are especially interested in cases that the matrix $A$ is symmetric positive definite or nonsymmetric with positive definite symmetric part (i.e., $A$ is real positive). When $A=A^{T}$, the linear system (1.1) is called the symmetric saddle point problem and, when $A \neq A^{T}$, it is called the nonsymmetric saddle point problem. According to Lemma 1.1 in [7] the matrix $\mathcal{A}$ is nonsingular.

In the last decade, there has been tremendous efforts to develop fast solution methods for solving the saddle point problems. As is well-known, Krylov subspace methods [15] are the most effective methods for solving the saddle point problems of the form (1.1). But the convergence rate of these methods depend closely on the eigenvalues and the eigenvectors of the

\footnotetext{
${ }^{*}$ Received August 28, 2016 / Revised version received May 31, 2017 / Accepted July 10, 2017 /

Published online August 21, 2018 /
} 
coefficient matrix $[1,15]$ and they tend to converge slowly when are applied to the saddle point problem (1.1). In general, favourable rates of convergence of Krylov subspace methods are often incorporated with a well-clustered spectrum of the preconditioned matrices (away from zero). Therefore, many kinds of preconditioners have been studied in the literature for saddle point matrix, e.g., HSS-based preconditioners [2,4,5,7], block diagonal preconditioners [17], block triangular preconditioners $[3,17]$, shift-splitting preconditioners [6,10], and so on.

Zhang and $\mathrm{Gu}$ in [18] established a variant of the deteriorated positive semi-definite and skew-Hermitian splitting (VDPSS) preconditioner as follows

$$
\mathcal{M}_{V D P S S}=\left(\begin{array}{cc}
A & \frac{1}{\alpha} A B^{T} \\
-B & \alpha I
\end{array}\right)
$$

for the problem (1.1). Recently, Huang et al. in [13] proposed a generalization of the VDPSS (GVDPSS) preconditioner of the form

$$
\mathcal{P}_{G V D P S S}=\left(\begin{array}{cc}
A & \frac{1}{\alpha} A B^{T} \\
-B & \beta I
\end{array}\right) .
$$

The difference between $\mathcal{P}_{G V D P S S}$ and $\mathcal{A}$ is given by

$$
\mathcal{R}_{G V D P S S}=\mathcal{P}_{G V D P S S}-\mathcal{A}=\left(\begin{array}{cc}
0 & \frac{1}{\alpha} A B^{T}-B^{T} \\
0 & \beta I
\end{array}\right) .
$$

It follows from the latter equation that as $\beta \longrightarrow 0^{+}$, the $(2,2)$-block of $\mathcal{R}_{G V D P S S}$ tends to zero matrix and as $\alpha \longrightarrow+\infty$, the $(1,2)$-block of $\mathcal{R}$ tends to $-B^{T}$. So, it seems that the GVDPSS preconditioner with proper parameters $\alpha$ and $\beta$ is more closer to the coefficient matrix $\mathcal{A}$ than the VDPSS preconditioner due to the independence of the parameters and, as a result, the corresponding preconditioned matrix will have a well-clustered spectrum.

It can be seen that by choosing different values for the parameters $\alpha$ and $\beta$, the GVDPSS preconditioner coincides with some existing preconditioners such as the RHSS preconditioner [11], the REHSS preconditioner [16], the RDPSS preconditioner [9] and the VDPSS preconditioner [18].

The GVDPSS preconditioner can be derived from the GVDPSS iteration method. Huang et al. have presented the convergence properties of the GVDPSS iteration method and the spectral properties of the corresponding preconditioned matrix in [13], but nothing about the optimal values of the involved parameters. In this paper, we present new convergence properties and the optimal parameters, which minimize the spectral radius of the iteration matrix of the GVDPSS iteration method.

\section{New Convergence Results for the GVDPSS Iteration Method}

The GVDPSS preconditioner $\mathcal{P}_{G V D P S S}$ can be induced by a fixed-point iteration, which is based on the following splitting of the coefficient matrix $\mathcal{A}$ :

$$
\mathcal{A}=\mathcal{P}_{G V D P S S}-\mathcal{R}_{G V D P S S}\left(\begin{array}{cc}
A & \frac{1}{\alpha} A B^{T} \\
-B & \beta I
\end{array}\right)-\left(\begin{array}{cc}
0 & \frac{1}{\alpha} A B^{T}-B^{T} \\
0 & \beta I
\end{array}\right) .
$$

Based on this splitting, the GVDPSS iteration method can be constructed as

$$
\left(\begin{array}{cc}
A & \frac{1}{\alpha} A B^{T} \\
-B & \beta I
\end{array}\right) u^{(k+1)}=\left(\begin{array}{cc}
0 & \frac{1}{\alpha} A B^{T}-B^{T} \\
0 & \beta I
\end{array}\right) u^{(k)}+\left(\begin{array}{c}
f \\
-g
\end{array}\right),
$$


where $\alpha>0$ and $\beta>0$ are the iteration parameters and $u^{(0)}$ is an initial guess. Note that the iterative method can also be written as a fixed point form $u^{k+1}=\Gamma u^{k}+c$, where

$$
\Gamma=\left(\begin{array}{cc}
A & \frac{1}{\alpha} A B^{T} \\
-B & \beta I
\end{array}\right)^{-1}\left(\begin{array}{cc}
0 & \frac{1}{\alpha} A B^{T}-B^{T} \\
0 & \beta I
\end{array}\right),
$$

is the iteration matrix and $c=\mathcal{P}^{-1} b$.

Theorem 2.1. Let $A \in \mathbb{R}^{n \times n}$ be symmetric positive definite and $\Gamma$ be the iteration matrix (2.3) of the iteration method $(2.2)$. Then, $\rho(\Gamma)<1$ if one of the following two conditions holds true.

(i) If $\alpha>0$ and $\beta \geq \max \left\{\lambda_{\max }(Q), 0\right\}$, where $\lambda_{\max }(Q)$ is the largest eigenvalue of the matrix $Q \equiv B\left(\frac{1}{2} A^{-1}-\frac{1}{\alpha} I\right) B^{T}$.

(ii) If $\beta \geq 0$ and $\alpha<2 \lambda_{\min }(A)$, where $\lambda_{\min }(A)$ is the smallest eigenvalue of $A$.

Proof. From Lemma 2.1 in [13], we get

$$
\begin{aligned}
& \Gamma=\mathcal{P}^{-1} \mathcal{R}=\mathcal{P}^{-1}(\mathcal{P}-\mathcal{A})=I-\mathcal{P}^{-1} \mathcal{A} \\
& =I-\left(\begin{array}{cc}
A^{-1}-\frac{1}{\alpha} B^{T} S^{-1} B A^{-1} & -\frac{1}{\alpha} B^{T} S^{-1} \\
S^{-1} B A^{-1} & S^{-1}
\end{array}\right)\left(\begin{array}{cc}
A & B^{T} \\
-B & 0
\end{array}\right) \\
& =I-\left(\begin{array}{cc}
I & A^{-1} B^{T}-\frac{1}{\alpha} B^{T} S^{-1} B A^{-1} B^{T} \\
0 & S^{-1} B A^{-1} B^{T}
\end{array}\right) \\
& =\left(\begin{array}{cc}
0 & \tilde{A} \\
0 & I-\hat{A}
\end{array}\right) \text {, }
\end{aligned}
$$

where $S=\beta I+\frac{1}{\alpha} B B^{T}, \tilde{A}=-A^{-1} B^{T}+\frac{1}{\alpha} B^{T} S^{-1} B A^{-1} B^{T}$ and $\hat{A}=S^{-1} B A^{-1} B^{T}$. Therefore, if $\lambda$ is an eigenvalue of the iteration matrix $\Gamma$, then $\lambda=0$ or $\lambda=1-\mu$, where $\mu$ is the solution of the eigenvalue problem

$$
S^{-1} B A^{-1} B^{T} x=\mu x .
$$

Since $S=\beta I+\frac{1}{\alpha} B B^{T}$, Eq. (2.5) is equivalent to

$$
B A^{-1} B^{T} x=\mu\left(\beta I+\frac{1}{\alpha} B B^{T}\right) x .
$$

It is clear that $x \neq 0$. Without loss of generality, we suppose that $\|x\|_{2}=1$. Since $B^{T} x \neq 0$, multiplying the above equation by $x^{*}$ on both sides yields

$$
\mu=\frac{x^{*} B A^{-1} B^{T} x}{\beta+\frac{1}{\alpha} x^{*} B B^{T} x}>0 .
$$

Therefore, $|\lambda|<1$ if and only if

$$
\frac{x^{*} B A^{-1} B^{T} x}{\beta+\frac{1}{\alpha} x^{*} B B^{T} x}<2
$$

which is equivalent to

$$
\beta>\frac{1}{2} x^{*} B A^{-1} B^{T} x-\frac{1}{\alpha} x^{*} B B^{T} x .
$$

To prove part (i), let $\alpha$ be an arbitrary positive constant. From above discussion, it is easy to know that a sufficient condition to have $|\lambda|<1$ is that

$$
\beta>\lambda_{\max }(Q)=\max _{\|y\|_{2}=1} y^{*} Q y>x^{*} Q x=\frac{1}{2} x^{*} B A^{-1} B^{T} x-\frac{1}{\alpha} x^{*} B B^{T} x,
$$


which completes the proof of (i).

Now, we prove the part (ii). If the right-hand side of the inequality (2.7) is less than zero then for every $\beta \geq 0$ the inequality (2.7) holds true and, as a result, we get $\rho(\Gamma)<1$. Hence, the parameter $\alpha$ must be chosen such a way that

$$
\frac{1}{2} x^{*} B A^{-1} B^{T} x-\frac{1}{\alpha} x^{*} B B^{T} x<0,
$$

which is equivalent to

$$
\alpha<\frac{2 y^{*} y}{y^{*} A^{-1} y}
$$

where $y=B^{T} x$. For the above relation to be held, it is enough to have

$$
\alpha<2 \lambda_{\min }(A)=\frac{2}{\lambda_{\max }\left(A^{-1}\right)}=2 \min _{y \neq 0} \frac{y^{*} y}{y^{*} A^{-1} y}<\frac{2 y^{*} y}{y^{*} A^{-1} y},
$$

which completes the proof.

Now, we consider the GVDPSS iteration method (2.2) from another point of view. Let $\omega \geq 0$ be a fixed constant. Consider the parameters $\alpha>0$ and $\beta \geq 0$ such that $\omega=\alpha \beta$. In this case, the iteration matrix $\Gamma$ of the GVDPSS iteration method (2.2) is given by

$$
\Gamma=\left(\begin{array}{cc}
0 & A^{-1} B^{T}-\frac{1}{\alpha} B^{T} S^{-1} B A^{-1} B^{T} \\
0 & I-\alpha\left(\omega I+B B^{T}\right)^{-1} B A^{-1} B^{T}
\end{array}\right)
$$

It follows from Eq. (2.9) that the spectral radius of the iteration matrix $\Gamma$ is

$$
\rho(\Gamma)=\max _{1 \leq i \leq m}\left|1-\alpha \mu_{i}\right|,
$$

where $\mu_{i}, i=1, \ldots, m$, are the eigenvalues of the matrix $\left(\omega I+B B^{T}\right)^{-1} B A^{-1} B^{T}$. Since $\left(\omega I+B B^{T}\right)^{-1} B A^{-1} B^{T}$ is similar to $\left(\omega I+B B^{T}\right)^{-\frac{1}{2}} B A^{-1} B^{T}\left(\omega I+B B^{T}\right)^{-\frac{1}{2}}$, then, in fact, the spectral radius (2.10) is the same as that of the stationary Richardson iteration when applied to the following linear system

$$
\left(\omega I+B B^{T}\right)^{-\frac{1}{2}} B A^{-1} B^{T}\left(\omega I+B B^{T}\right)^{-\frac{1}{2}} x=b .
$$

Therefore, it is expected that the convergence properties of the Richardson iteration method and the GVDPSS iteration method (2.2) with $\beta=\omega / \alpha$ are the same. Then, using the above results and an argument like Theorem 3.1 in [11], we can deduce the following theorem.

Theorem 2.2. Let $A \in \mathbb{R}^{n \times n}$ be symmetric positive definite and $\Gamma$ be the iteration matrix (2.3) of the GVDPSS iteration method (2.2). Let $\omega \geq 0$ be a constant and consider the parameters $\alpha>0$ and $\beta \geq 0$ such that $\omega=\alpha \beta$. Then,

$$
\rho(\Gamma)=\max _{1 \leq i \leq m}\left|1-\alpha \mu_{i}\right|
$$

where $\mu_{i}, i=1, \ldots, m$, are the eigenvalues of $\left(\omega I+B B^{T}\right)^{-1} B A^{-1} B^{T}$. Let $\mu_{m}$ and $\mu_{1}$ be the smallest and largest eigenvalues of $\left(\omega I+B B^{T}\right)^{-1} B A^{-1} B^{T}$, respectively. For every $\omega \geq 0$, if

$$
0<\alpha<\frac{1}{\mu_{1}},
$$


and $\beta=\omega / \alpha$ then the GVDPSS iteration method is convergent. The optimal value of $\alpha$ which minimizes the spectral radius $\rho(\Gamma)$ is given by $\alpha_{\mathrm{opt}}=2 /\left(\mu_{1}+\mu_{m}\right)$, which yields $\beta_{\mathrm{opt}}=\omega / \alpha_{\mathrm{opt}}$. The corresponding optimal convergence factor is given by

$$
\rho(\Gamma)=\frac{\mu_{1}-\mu_{m}}{\mu_{1}+\mu_{m}}
$$

Remark 2.1. If $\omega=0$ then $\beta=0$ and, as a result, the preconditioner $\mathcal{P}_{G V D P S S}$ reduces to the RHSS preconditioner. Hence, the corresponding optimal value $\alpha^{*}$ is exactly the same as that of the RHSS preconditioner (Theorem 3.1 in [11]).

Theorem 2.3. Let $A \in \mathbb{R}^{n \times n}$ be symmetric positive definite, the preconditioner $\mathcal{P}_{G V D P S S}$ be defined in (1.3) and $\alpha>0$ and $\beta \geq 0$. Let $\lambda_{\min }$ and $\lambda_{\max }$ be the smallest and largest eigenvalues of $\mathcal{A}$, respectively. Let $\sigma_{m}$ and $\sigma_{1}$ be the smallest and largest singular values of matrix $B$, respectively. Then, the preconditioned matrix $\mathcal{P}_{G V D P S S}^{-1} \mathcal{A}$ has an eigenvalue 1 of algebraic multiplicity at least $n$. The remaining eigenvalues are positive real and the solution of the generalized eigenvalue problem:

$$
B A^{-1} B^{T} x=\mu\left(\beta I+\frac{1}{\alpha} B B^{T}\right) x .
$$

In addition, the non-unit eigenvalues $\mu$ satisfy

$$
\frac{\alpha}{\lambda_{\max }(A)(1+\alpha \beta)} \leq \mu \leq \frac{\alpha}{\lambda_{\min }(A)(1+\alpha \beta)} .
$$

Proof. It follows from Eq. (2.4) that

$$
\mathcal{P}^{-1} \mathcal{A}=\left(\begin{array}{cc}
I & A^{-1} B^{T}-\frac{1}{\alpha} B^{T} S^{-1} B A^{-1} B^{T} \\
0 & S^{-1} B A^{-1} B^{T}
\end{array}\right)=\left(\begin{array}{cc}
I & \tilde{A} \\
0 & \hat{A}
\end{array}\right)
$$

where $S=\beta I+\frac{1}{\alpha} B B^{T}$. It follows from Eq. (2.12) that the preconditioned matrix $\mathcal{P}_{G V D P S S}^{-1} \mathcal{A}$ has an eigenvalue 1 of algebraic multiplicity at least $n$, and the remaining eigenvalues are the solution of the following generalized eigenvalue problem

$$
B A^{-1} B^{T} x=\mu\left(\beta I+\frac{1}{\alpha} B B^{T}\right) x .
$$

Since $B B^{T}$ and $B A^{-1} B^{T}$ are symmetric and positive definite, $\beta \geq 0$ and $\alpha>0$, the solution of the generalized eigenvalue problem (2.13) is positive real. This completes the proof of the first part of the theorem. Let $(\mu, x)$ be an eigenpair of the generalized eigenvalue problem (2.13). If we set $\hat{x}=x /\left\|B^{T} x\right\|_{2}$ then $(\mu, \hat{x})$ is also an eigenpair of (2.13). As seen in the proof of Theorem 2.1 , we can write

$$
\mu=\frac{\hat{x}^{*} B A^{-1} B^{T} \hat{x}}{\beta+\frac{1}{\alpha} \hat{x}^{*} B B^{T} \hat{x}}=\frac{\hat{x}^{*} B A^{-1} B^{T} \hat{x}}{\beta+\frac{1}{\alpha}} .
$$

According to Theorem 1.22 of [15], we have

$$
\begin{aligned}
& \hat{x}^{*} B A^{-1} B^{T} \hat{x} \leq \lambda_{\max }\left(A^{-1}\right) \hat{x}^{*} B B^{T} \hat{x}=\frac{1}{\lambda_{\min }(A)}, \\
& \frac{1}{\lambda_{\max }(A)}=\lambda_{\min }\left(A^{-1}\right) \hat{x}^{*} B B^{T} \hat{x} \leq \hat{x}^{*} B A^{-1} B^{T} \hat{x},
\end{aligned}
$$

that prove (2.11). 
Remark 2.2. It immediately follows from Eq. (2.11) that if $\beta \longrightarrow 0^{+}$then the non-unit eigenvalues $\mu$ of the preconditioned matrix $\mathcal{P}^{-1} \mathcal{A}$ satisfy

$$
\frac{\alpha}{\lambda_{\max }(A)} \leq \mu \leq \frac{\alpha}{\lambda_{\min }(A)}
$$

and if $\alpha \longrightarrow+\infty$, then

$$
\frac{1}{\beta \lambda_{\max }(A)} \leq \mu \leq \frac{1}{\beta \lambda_{\min }(A)} .
$$

Theorem 2.4. Let $A \in \mathbb{R}^{n \times n}$ be nonsymmetric and positive definite and $\Gamma$ be the iteration matrix (2.3) of the GVDPSS iteration method (2.2). Let $\omega \geq 0$ be a fixed constant and consider the parameters $\alpha>0$ and $\beta \geq 0$ such that $\omega=\alpha \beta$. Then,

$$
\rho(\Gamma)=\max _{1 \leq j \leq m}\left|1-\alpha\left(\gamma_{j}+i \eta_{j}\right)\right|
$$

where $\gamma_{j}+i \eta_{j}, j=1, \ldots, m$, are the eigenvalues of $\left(\omega I+B B^{T}\right)^{-1} B A^{-1} B^{T}$ and $i=\sqrt{-1}$. Let $\gamma_{1}$ and $\gamma_{m}$, and $\eta_{1}$ and $\eta_{m}$ be the upper and lower bounds of the real, the absolute values of the imaginary parts of the eigenvalues of $\left(\omega I+B B^{T}\right)^{-1} B A^{-1} B^{T}$, respectively. For every $\omega \geq 0$, if

$$
0<\alpha< \begin{cases}\frac{2 \gamma_{m}}{\gamma_{m}^{2}+\eta_{1}^{2}}, & \text { if } \eta_{1} \geq \sqrt{\gamma_{1} \gamma_{m}} \\ \frac{2 \gamma_{1}}{\gamma_{1}^{2}+\eta_{1}^{2}}, & \text { if } \eta_{1}<\sqrt{\gamma_{1} \gamma_{m}}\end{cases}
$$

and $\beta=\omega / \alpha$ then the GVDPSS iteration method is convergent. The optimal value of $\alpha$ which minimizes the spectral radius $\rho(\Gamma)$ is given by

$$
\alpha_{\mathrm{opt}}= \begin{cases}\frac{\gamma_{m}}{\gamma_{m}^{2}+\eta_{1}^{2}}, & \text { if } \eta_{1} \geq \sqrt{\gamma_{1} \gamma_{m}}, \\ \frac{2}{\gamma_{1}+\gamma_{m}}, & \text { if } \eta_{1}<\sqrt{\gamma_{1} \gamma_{m}},\end{cases}
$$

which yields $\beta_{\mathrm{opt}}=\omega / \alpha_{\mathrm{opt}}$. The corresponding optimal convergence factor is given by

$$
\rho_{\mathrm{opt}}(\gamma)= \begin{cases}\frac{\eta_{1}}{\sqrt{\gamma_{m}^{2}+\eta_{1}^{2}}}, & \text { if } \eta_{1} \geq \sqrt{\gamma_{1} \gamma_{m}}, \\ \frac{\sqrt{\left(\gamma_{1}-\gamma_{m}\right)^{2}+4 \eta_{1}^{2}}}{\gamma_{1}+\gamma_{m}}, & \text { if } \eta_{1}<\sqrt{\gamma_{1} \gamma_{m}}\end{cases}
$$

Proof. As seen before, when $\beta=\omega / \alpha$, the iteration matrix $\Gamma$ is of the form (2.9). It shows that the spectral radius of the iteration matrix $\Gamma$ is

$$
\rho(\Gamma)=\max _{1 \leq j \leq m}\left|1-\alpha\left(\gamma_{j}+i \eta_{j}\right)\right|
$$

where $\gamma_{j}+i \eta_{j}, j=1, \ldots, m$, are the eigenvalues of $\left(\omega I+B B^{T}\right)^{-1} B A^{-1} B^{T}$. The rest of the proof is similar to that of Theorem 2.2 in [9] and is omitted.

Remark 2.3. If $\omega=0$, then $\beta=0$ and, as a result, the preconditioner $\mathcal{P}_{G V D P S S}$ reduces to the RDPSS preconditioner. Hence, the corresponding optimal value $\alpha^{*}$ is exactly the same as that of the RDPSS preconditioner (Theorem 2.2 in [9]). 

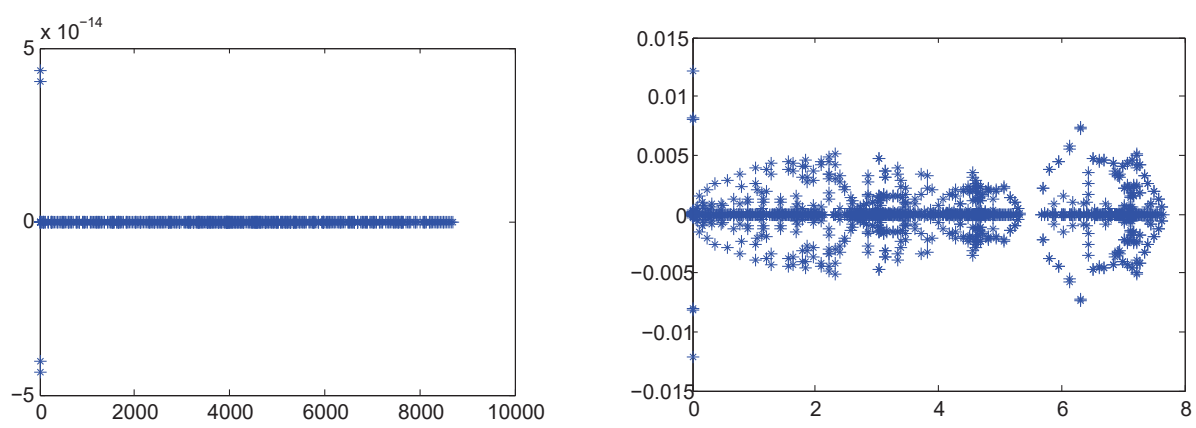

Fig. 3.1. Eigenvalue distribution of the saddle point matrix $\mathcal{A}$ for Example 3.1 with $q=32$ (left) and for Example 3.2 with $32 \times 32$ grid (right).

\section{Numerical Experiments}

In this section, we use two examples to validate the theoretical analysis of the previous section and illustrate the feasibility and effectiveness of the GVDPSS preconditioner (1.3) when it is applied to accelerate the convergence rate of Krylov subspace iteration methods such as GMRES for solving saddle point problems (1.1), from the point of view of both the number of iterations (denoted by IT) and and the elapsed CPU time in seconds (denoted by CPU). As mentioned before, the GVDPSS preconditioner (1.3) covers the RHSS preconditioner [11] and the REHSS preconditioner [16] when it is applied to solve the symmetric saddle point problems as well as the DPSS preconditioner [14] and the VDPSS preconditioner [18] when it is applied to solve the nonsymmetric saddle point problems. The first and second examples lead to a symmetric and nonsymmetric saddle point problem, respectively. The Krylov subspace methods such as GMRES incorporated with RHSS preconditioner, REHSS preconditioner, the GVDPSS preconditioner, the PHSS preconditioner [5] and the AHSS preconditioner [2] are applied to solve the symmetric saddle point problem in the first example, and then their results are compared with each other. In addition, the RPDSS and VDPSS preconditioners as well as the GVDPSS preconditioner are applied to solve the nonsymmetric saddle point problem in the second example, and then their results are compared with each other.

All tests are performed in MATLAB on a Laptop with Intel Core i7 CPU $1.8 \mathrm{GHz}, 6 \mathrm{~GB}$ RAM. In all the tests, the right-hand side vector $b$ is chosen so that the exact solution of the saddle point problem (1.1) is a vector of all ones. Besides, all runs are started from a null vector, and are terminated if the current iteration satisfies $\left\|r_{k}\right\|_{2} \leq 10^{-6}\left\|r_{0}\right\|_{2}$, where $r_{k}=b-\mathcal{A} u^{(k)}$ is the residual at the $k$ th iteration. At each step of applying the preconditioners, it is required to solve the sub-linear systems which can be done by direct methods. In MATLAB, this corresponds to computing the Cholesky or LU factorization in combination with AMD or column AMD reordering.

Example 3.1. ([5]) We consider the Stokes problem

$$
\begin{array}{ll}
-\mu \triangle \mathbf{u}+\nabla p=\tilde{f}, & \text { in } \Omega, \\
\nabla \cdot \mathbf{u}=\tilde{g}, & \text { in } \Omega, \\
\mathbf{u}=0, & \text { on } \partial \Omega, \\
\int_{\Omega} p d x=0, &
\end{array}
$$



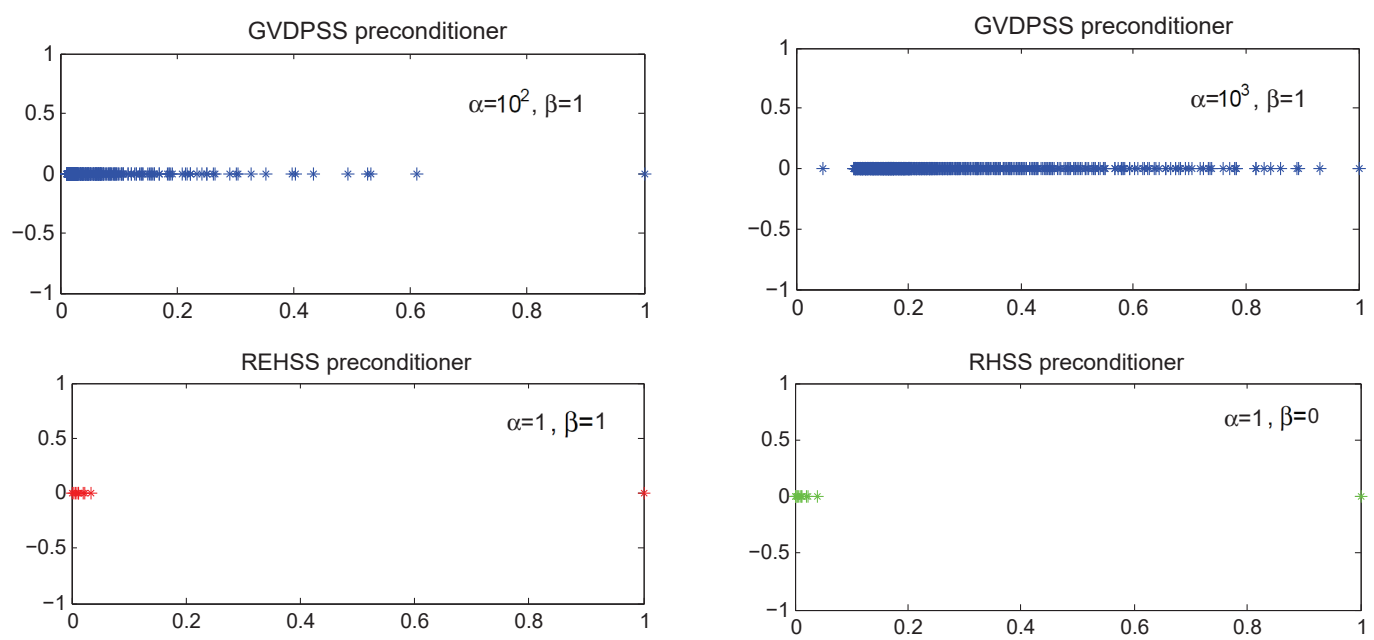

Fig. 3.2. Eigenvalue distribution of the preconditioned matrix for Example $3.1(q=32)$.
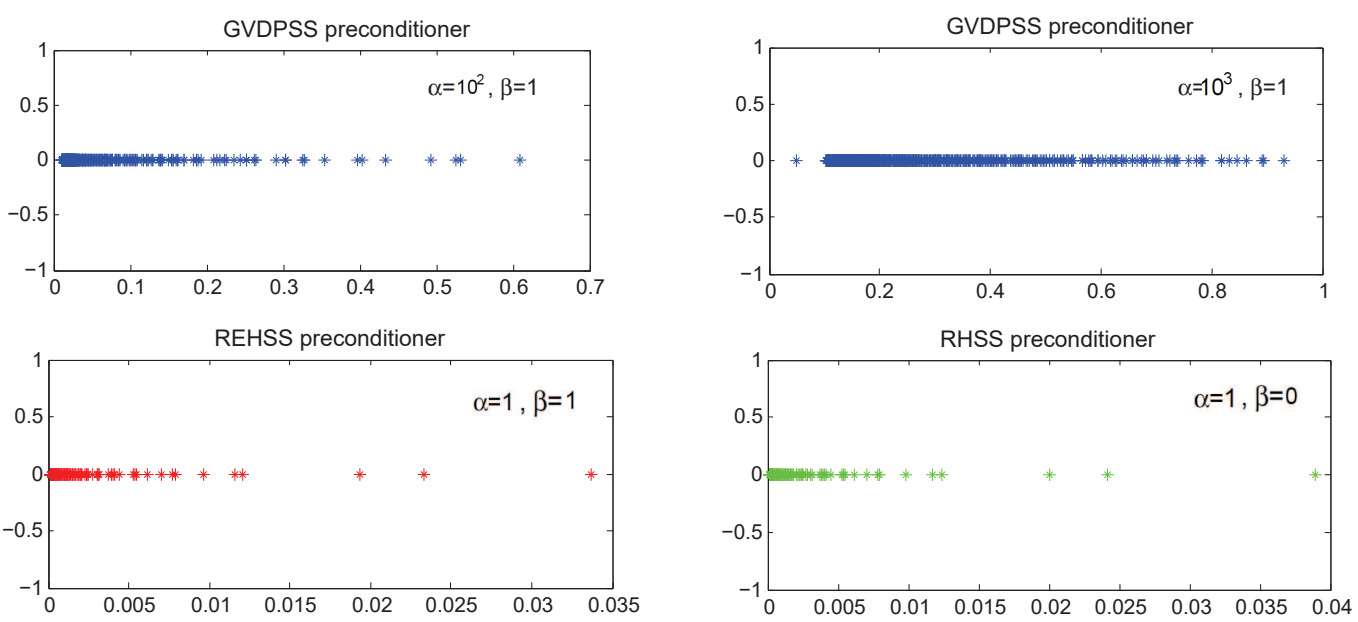

Fig. 3.3. Non-unit eigenvalue distribution of the preconditioned matrix for Example $3.1(q=32)$.

where $\Omega=(-1,1) \times(-1,1), \partial \Omega$ is the boundary of the domain $\Omega, \triangle$ is the componentwise Laplace operator, $\mathbf{u}$ is a vector-valued function representing the velocity, and $p$ is a scalar function representing the pressure. By discretizing (3.1) by the upwind scheme, we obtain the system of linear equations (1.1) where

$$
\begin{aligned}
& A=\left(\begin{array}{cc}
I \otimes T+T \otimes I & 0 \\
0 & I \otimes T+T \otimes I
\end{array}\right) \in \mathbb{R}^{2 q^{2} \times 2 q^{2}}, \quad B^{T}=\left(\begin{array}{c}
I \otimes F \\
F \otimes I
\end{array}\right) \in \mathbb{R}^{2 q^{2} \times q^{2}}, \\
& T=\frac{\mu}{h^{2}} \cdot \operatorname{tridiag}(-1,2,-1) \in \mathbb{R}^{q \times q}, \quad F=\frac{1}{h} \cdot \operatorname{tridiag}(-1,1,0) \in \mathbb{R}^{q \times q},
\end{aligned}
$$

with $\otimes$ being the Kronecker product symbol and $h=1 /(q+1)$ the discretization meshsize. For this example, we have $n=2 q^{2}$ and $m=q^{2}$. Hence, the total number of variables is $m+n=3 q^{2}$. Here, we consider $\mu=1$. 

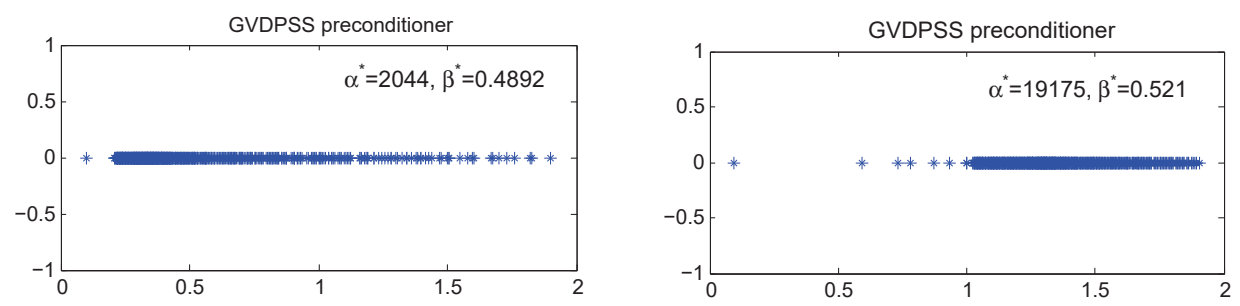

Fig. 3.4. Eigenvalue distribution of the preconditioned matrix with optimal parameters for Example $3.1(q=32)$.

From [2], we know that the AHSS preconditioner is given by

$$
\mathcal{M}(\alpha, \beta)=\left(\begin{array}{cc}
\frac{\alpha+1}{2} A & \frac{\alpha+1}{2 \alpha} B^{T} \\
-\frac{1}{2} B & \frac{\beta}{2} C
\end{array}\right),
$$

where $C$ is an arbitrary Hermitian positive definite matrix. It is noted that the PHSS preconditioner is a special case of the AHSS preconditioner when $\alpha=\beta$. A natural choice of the matrix $C$ is $C=B \widehat{A}^{-1} B^{T}$, where $\widehat{A}$ is a good approximation of the matrix block $A$. For this example, we take $\widehat{A}=\frac{2 \mu}{h^{2}} I+I \otimes T$ the block-diagonal matrix $A$. Based on Theorem 3.2 in [5] and [2], we can obtain the optimal parameters $\alpha^{*}$ and $\left(\alpha^{*}, \beta^{*}\right)$ of the PHSS and AHSS iteration methods, respectively. In Table 3.1, these optimal parameters and the corresponding numerical results with respect to iteration step and CPU time, for various problem sizes $q$, are listed. In Table 3.2, we list the optimal parameters $\alpha^{*}$ and $\beta^{*}$ of the GVDPSS preconditioner, determined by the formula given in Theorem 2.2, and the corresponding numerical results with respect to iteration step and CPU time for different values of $\omega$ and $q$. For each $q$, it is seen that by changing $\omega$, the changes of the corresponding optimal parameter $\beta^{*}$ in comparison with optimal parameter $\alpha^{*}$ is significantly slow (for new preconditioner). It should be noted that if $\omega=0$ then $\beta=0$ and, as a result, the preconditioner $\mathcal{P}_{G V D P S S}$ reduces to the RHSS preconditioner which its results were shown in boldface type. It is also noticed that $\alpha^{*}$ corresponding to $\omega=0$ plays the role of the optimal parameter of the RHSS preconditioner. As seen, the preconditioner $\mathcal{P}_{G V D P S S}$ with optimal parameters is more effective than the RHSS, PHSS and AHSS preconditioners in terms of both the iteration steps and the CPU time. Moreover, by increasing the value of $\omega$, the preconditioner $\mathcal{P}_{G V D P S S}$ with corresponding optimal parameters has a considerable reduction in the number of the iteration steps.

To further investigate the influence of the new parameter in the convergence speed of the

Table 3.1: The optimal parameters of the PHSS and AHSS preconditioners and the corresponding numerical results for Example 3.1 with different $q$.

\begin{tabular}{|llllll|}
\hline Method & $q$ & 16 & 32 & 48 & 64 \\
\hline PHSS-GMRES & $\alpha^{*}$ & 1.8718 & 2.5657 & 3.1113 & 3.5753 \\
& IT & 46 & 94 & 149 & 209 \\
& CPU & 0.106 & 5.049 & 51.01 & 266 \\
AHSS-GMRES & $\alpha^{*}$ & 1.5026 & 1.9482 & 2.3115 & 2.6256 \\
& $\beta^{*}$ & 2.3317 & 3.3789 & 4.1879 & 4.8685 \\
& IT & 43 & 84 & 131 & 184 \\
& CPU & 0.097 & 4.424 & 43.57 & 235 \\
\hline
\end{tabular}


Table 3.2: The optimal parameters of the GVDPSS preconditioner and the numerical results for the corresponding preconditioned GMRES method for Example 3.1 with different $\omega$ and $q$.

\begin{tabular}{|c|ccccc|}
\hline$q$ & $\omega$ & $\alpha^{*}$ & $\beta^{*}$ & IT & CPU \\
\hline \multirow{6}{*}{16} & $\mathbf{0}$ & $\mathbf{4 9 . 2 5}$ & $\mathbf{0}$ & $\mathbf{2 3}$ & $\mathbf{0 . 0 1 7 8}$ \\
& 1 & 56.91 & 0.0176 & 23 & 0.01795 \\
& $10^{1}$ & 104.32 & 0.0959 & 21 & 0.01602 \\
& $10^{2}$ & 307.61 & 0.3251 & 15 & 0.01195 \\
& $10^{3}$ & 1966 & 0.5086 & 10 & 0.0094 \\
& $10^{4}$ & 18473 & 0.5413 & 9 & 0.0087 \\
\hline \multirow{6}{*}{32} & $\mathbf{0}$ & $\mathbf{5 1 . 1 9}$ & $\mathbf{0}$ & $\mathbf{3 6}$ & $\mathbf{0 . 1 1 5 1}$ \\
& 1 & 59.18 & 0.0169 & 36 & 0.1097 \\
& $10^{1}$ & 107.34 & 0.0932 & 34 & 0.10290 \\
& $10^{2}$ & 321.8 & 0.3108 & 26 & 0.0769 \\
& $10^{3}$ & 2044 & 0.4892 & 15 & 0.0470 \\
& $10^{4}$ & 19175 & 0.521 & 10 & 0.0343 \\
\hline \multirow{6}{*}{48} & $\mathbf{0}$ & $\mathbf{5 1 . 8 2}$ & $\mathbf{0}$ & $\mathbf{4 7}$ & $\mathbf{0 . 5 2 1 0}$ \\
& 1 & 59.90 & 0.0167 & 46 & 0.5153 \\
& $10^{1}$ & 108.06 & 0.0925 & 44 & 0.4892 \\
& $10^{2}$ & 324.5 & 0.3081 & 36 & 0.3931 \\
& $10^{3}$ & 2076 & 0.4817 & 19 & 0.2146 \\
& $10^{4}$ & 19461 & 0.5138 & 11 & 0.1338 \\
\hline \multirow{6}{*}{64} & $\mathbf{0}$ & $\mathbf{5 2 . 1 3}$ & $\mathbf{0}$ & $\mathbf{5 6}$ & $\mathbf{1 . 9 5 0}$ \\
& 1 & 60.25 & 0.0166 & 56 & 1.926 \\
& $10^{1}$ & 108.36 & 0.0923 & 54 & 1.884 \\
& $10^{2}$ & 325.48 & 0.3072 & 45 & 1.561 \\
& $10^{3}$ & 2093 & 0.4776 & 23 & 0.799 \\
& $10^{4}$ & 19616 & 0.5098 & 11 & 0.406 \\
\hline \multirow{6}{*}{} & & & & & \\
& & & & & \\
& & & \\
& & &
\end{tabular}

preconditioned GMRES method, we present the numerical results for different values of $\alpha$ and $\beta$ in Table 3.3 with respect to iteration step and CPU time (in parentheses). It should be noted that when $\beta=0$ the preconditioner $\mathcal{P}_{G V D P S S}$ reduces to the RHSS preconditioner which its results were shown in boldface type, and when $\alpha=1$ it coincides with the REHSS preconditioner which its results were underlined. Table 3.3 indicates that the GVDPSS preconditioner is more effective than the RHSS, REHSS, PHSS and AHSS preconditioners. Specially, for $\beta \geq 1$, the use of the GVDPSS preconditioner with some large values of $\alpha$ results in a considerable reduction in the number of iteration steps.

We plot the eigenvalue distribution of the matrix $\mathcal{A}$ in Fig 3.1 and that of the preconditioned matrix $\mathcal{P}_{G V D P S S}^{-1} \mathcal{A}$ in Fig 3.2. As mentioned, if $\beta=0$ then the $\mathcal{P}_{G V D P S S}$ preconditioner reduces to the RHSS preconditioner and if $\alpha=1$ then the $\mathcal{P}_{G V D P S S}$ preconditioner reduces to the REHSS preconditioner. The non-unit eigenvalue distribution of the preconditioned matrices in Fig 3.2 are drawn in Fig 3.3. Fig 3.4 depicts the eigenvalue distribution of the preconditioned matrix $\mathcal{P}_{G V D P S S}^{-1} \mathcal{A}$ with optimal parameters. These figures show that the use of the preconditioner $\mathcal{P}_{G V D P S S}$ leads to a well-clustered spectrum away from zero which in turn results in a faster convergence of GMRES. In addition, Fig 3.2 verifies our statement in Theorem 2.3 that the preconditioned matrix $\mathcal{P}_{G V D P S S}^{-1} \mathcal{A}$ has at least $n$ eigenvalues 1 and the remaining non-unit eigenvalues are positive real.

Example 3.2. Consider the Oseen problem which is obtained from the linearization of the following steady-state Navier-Stokes equation by the Picard iteration with suitable boundary 
Table 3.3: Numerical results for the GMRES method incorporated with the GVDPSS preconditioner for Example 3.1 with different $q$.

\begin{tabular}{|c|c|c|c|c|c|c|}
\hline$q$ & $\alpha$ & 0 & $10^{-1}$ & 1 & 10 & $10^{2}$ \\
\hline \multirow{6}{*}{16} & $10^{-1}$ & $27(0.0189)$ & $27(0.0199)$ & $27(0.0184)$ & $27(0.0193)$ & $26(0.0199)$ \\
\hline & 1 & $25(0.0178)$ & $25(0.0193)$ & $25(0.0182)$ & $24(0.0191)$ & $19(0.0145)$ \\
\hline & 10 & $\overline{24(0.0170)}$ & $\overline{24(0.0166)}$ & $\overline{23(0.0163)}$ & $\overline{18(0.0142)}$ & $\overline{11(0.0099)}$ \\
\hline & $10^{2}$ & $22(0.0160)$ & $21(0.0150)$ & $16(0.0125)$ & $11(0.0096)$ & $9(0.0085)$ \\
\hline & $10^{3}$ & $21(0.0166)$ & $15(0.0122)$ & $9(0.0086)$ & $7(0.0077)$ & $8(0.0081)$ \\
\hline & $\alpha$ & 0 & $10^{-1}$ & 1 & 10 & $10^{2}$ \\
\hline \multirow{6}{*}{32} & $10^{-1}$ & $43(0.1393)$ & $43(0.1393)$ & $43(0.1359)$ & $43(0.1336)$ & $\overline{41(0.1263)}$ \\
\hline & 1 & $41(0.1243)$ & $41(0.1243)$ & $41(0.1242)$ & $39(0.1230)$ & $32(0.0994)$ \\
\hline & 10 & $\overline{38(0.1142)}$ & $\overline{38(0.1142)}$ & $\overline{37(0.1176)}$ & $\overline{30(0.1014)}$ & $\overline{18(0.0578)}$ \\
\hline & $10^{2}$ & $34(0.1059)$ & $34(0.1059)$ & $28(0.0866)$ & $16(0.0515)$ & $10(0.0369)$ \\
\hline & $10^{3}$ & $26(0.0811)$ & $26(0.0811)$ & $15(0.0557)$ & $10(0.0362)$ & $8(0.0291)$ \\
\hline & $\alpha$ & 0 & $10^{-1}$ & 1 & 10 & $10^{2}$ \\
\hline \multirow{6}{*}{48} & $10^{-1}$ & $56(0.6560)$ & $56(0.6456)$ & $56(0.7553)$ & $56(0.7061)$ & $54(0.6389)$ \\
\hline & 1 & $53(0.6176)$ & 53(0.6288) & $53(0.6974)$ & $51(0.6233)$ & $44(0.5325)$ \\
\hline & 10 & $\overline{50(0.5898)}$ & $\overline{50(0.6024)}$ & $\overline{48(0.5910)}$ & $\overline{42(0.5024)}$ & $\overline{23(0.3170)}$ \\
\hline & $10^{2}$ & $46(0.5609)$ & $45(0.5408)$ & $38(0.4549)$ & $22(0.2665)$ & $11(0.1349)$ \\
\hline & $10^{3}$ & $44(0.5493)$ & $36(0.4043)$ & $19(0.2428)$ & $11(0.1366)$ & $9(0.1194)$ \\
\hline & $\alpha$ & 0 & $10^{-1}$ & 1 & 10 & $10^{2}$ \\
\hline \multirow{5}{*}{64} & $10^{-1}$ & $68(2.550)$ & $68(2.495)$ & $68(2.488)$ & $68(2.501)$ & $66(2.449)$ \\
\hline & 1 & $64(2.334)$ & $64(2.347)$ & $64(2.340)$ & $62(2.277)$ & $54(1.962)$ \\
\hline & 10 & $\overline{60(2.154)}$ & $\overline{60(2.162)}$ & $\overline{58(2.119)}$ & $\overline{51(1.842)}$ & $\overline{28(1.005)}$ \\
\hline & $10^{2}$ & $55(1.962)$ & $54(1.952)$ & $48(1.743)$ & $26(0.919)$ & $13(0.484)$ \\
\hline & $10^{3}$ & $53(1.921)$ & $44(1.590)$ & $23(0.840)$ & $12(0.444)$ & $9(0.347)$ \\
\hline
\end{tabular}

condition on $\partial \Omega$

$$
\begin{cases}-\nu \triangle \mathbf{u}+(\mathbf{w} \cdot \nabla) \mathbf{u}+\nabla p=f, & \text { in } \Omega, \\ \operatorname{div} \mathbf{u}=0, & \text { in } \Omega, \\ \mathbf{u}=g, & \text { on } \partial \Omega\end{cases}
$$

where $\nu>0, \triangle, \nabla$, div, $\mathbf{u}$, and $p$ stand for the Laplace operator, the gradient operator, the divergence, velocity and pressure of the fluid, respectively. Here the vector field $\mathbf{w}$ is the approximation of $\mathbf{u}$ from the previous Picard iteration.

Many approximation schemes can be applied to discretize the Oseen problem (3.2) leading to a saddle point system of type (1.1). We consider a leaky two-dimensional lid-driven cavity problem discretized by Q2-P1 finite element on uniform grids on the unit square. The test problem was generated by using the IFISS software package written by Elman et al. [12]. We use the viscosity value $\nu=1$ to generate linear systems corresponding to $16 \times 16,32 \times 32,64 \times 64$ and $128 \times 128$ meshes. The numerical results for optimal parameters $\alpha^{*}$ and $\beta^{*}$, determined by the formula given in Theorem 2.4, corresponding to different valuses of $\omega$ on $16 \times 16,32 \times 32$, $64 \times 64$ uniform grids are given in Table 3.4. For each grid, we can see that by changing $\omega$, the corresponding optimal values $\beta^{*}$ is almost constant while the optimal values $\alpha^{*}$ changes increasingly. In addition, it is observed that the values of optimal parameters of the grid $32 \times 32$ 
Table 3.4: The optimal parameters of the GVDPSS preconditioner and the numerical results for the corresponding preconditioned GMRES method for Example 3.2 with different uniform grids.

\begin{tabular}{|c|ccccc|}
\hline Grids & $\omega$ & $\alpha^{*}$ & $\beta^{*}$ & IT & CPU \\
\hline \multirow{5}{*}{$16 \times 16$} & $\mathbf{0}$ & $\mathbf{0 . 1 9 0 9}$ & $\mathbf{0}$ & $\mathbf{2 7}$ & $\mathbf{0 . 0 5 5 7}$ \\
& 0.1 & 3.846 & 0.0260 & 20 & 0.0396 \\
& 1 & 33.018 & 0.0303 & 20 & 0.0397 \\
& 5 & 161.28 & 0.0310 & 20 & 0.0399 \\
& 10 & 321.13 & 0.0311 & 20 & 0.0401 \\
\hline \multirow{5}{*}{$32 \times 32$} & $\mathbf{0}$ & $\mathbf{0 . 0 4 8 8}$ & $\mathbf{0}$ & $\mathbf{4 2}$ & $\mathbf{0 . 2 7 7 8}$ \\
& 0.1 & 13.156 & 0.008 & 21 & 0.1631 \\
& 1 & 128.54 & 0.008 & 20 & 0.1452 \\
& 5 & 640 & 0.008 & 20 & 0.1491 \\
& 10 & 1280 & 0.008 & 20 & 0.1517 \\
\hline \multirow{5}{*}{$64 \times 64$} & $\mathbf{0}$ & $\mathbf{0 . 0 1 2 3}$ & $\mathbf{0}$ & $\mathbf{6 6}$ & $\mathbf{2 . 8 9 3}$ \\
& 0.1 & 14.339 & 0.007 & 20 & 0.925 \\
& 1 & 138.04 & 0.007 & 20 & 0.933 \\
& 5 & 689 & 0.007 & 20 & 0.933 \\
& 10 & 1379 & 0.007 & 20 & 0.906 \\
\hline
\end{tabular}

are significantly close to that of the grid size $64 \times 64$. It should be noted that if $\omega=0$ then $\beta=0$ and, as a result, the preconditioner $\mathcal{P}_{\text {GVDPSS }}$ reduces to the RDPSS preconditioner which its results were shown in boldface type. It is also noticed that $\alpha^{*}$ corresponding to $\omega=0$ plays the role of the optimal parameter of the RDPSS preconditioner. As seen, the performance of the preconditioner $\mathcal{P}_{G V D P S S}$ with optimal parameters is better than that of the RDPSS preconditioner in terms of both iteration steps and CPU time. Moreover, it is known that the efficiency of the preconditioner $\mathcal{P}_{G V D P S S}$ with optimal parameters corresponding to nonzero $\omega$ is often the same.

Further, in Table 3.5, we present the numerical results with respect to iteration step and CPU time (in parentheses) for different values of $\alpha$ and $\beta$ to analyze the influence of the GVDPSS parameter in the convergence speed of the preconditioned GMRES method. It should be noted that when $\beta=0$ the preconditioner $\mathcal{P}_{G V D P S S}$ reduces to the RDPSS preconditioner which its results were shown in boldface type, and when $\alpha=\beta$ it reduces to the VRDPSS preconditioner which its results were underlined. This table indicates that the performance of the new preconditioner $\mathcal{P}_{G V D P S S}$ is often better than that of the RDPSS and VRDPSS preconditioners. Specially in the case of small values of $\beta$, the use of preconditioner $\mathcal{P}_{G V D P S S}$ with some large value of $\alpha$ results in a considerable reduction in the number of iteration steps, while the other two preconditioners are difficult to implement efficiently. For large value of $\beta$, the GVDPSS preconditioner has the best performance and is no longer sensitive to the value of the parameter $\alpha$, in the sense that the number of iteration does not change drastically. It is also noted that the GVDPSS preconditioner is not competitive in the case of large $\beta$.

We plot the eigenvalue distribution of the matrix $\mathcal{A}$ in Fig 3.1, the eigenvalue distribution of the preconditioned matrix $\mathcal{P}_{G V D P S S}^{-1} \mathcal{A}$ in Fig 3.5, and the eigenvalue distribution of the preconditioned matrix $\mathcal{P}_{G V D P S S}^{-1} \mathcal{A}$ with optimal parameters in Fig 3.6. As mentioned, if $\beta=0$ then the preconditioner $\mathcal{P}_{G V D P S S}$ reduces to the RDPSS preconditioner and if $\alpha=\beta$ then the preconditioner $\mathcal{P}_{G V D P S S}$ reduces to the VDPSS preconditioner. It is evident that the preconditioner matrix $\mathcal{P}_{G V D P S S}^{-1} \mathcal{A}$ is of a well-clustered spectrum around $(1,0)$ away from zero. In addition, these figures verify the our statement in Theorem 2.3 that the preconditioned 
Table 3.5: Numerical results for the GMRES method incorporated with the GVDPSS preconditioner for Example 3.2 with different uniform grids.

\begin{tabular}{|c|c|c|c|c|c|c|c|}
\hline Grids & & 0 & $10^{-3}$ & $10^{-2}$ & $10^{-1}$ & 1 & $10^{2}$ \\
\hline \multirow{6}{*}{$16 \times 16$} & $10^{-3}$ & $31(0.0579)$ & $31(0.0541)$ & $31(0.0511)$ & $31(0.0531)$ & $30(0.0568)$ & $26(0.0437)$ \\
\hline & $10^{-2}$ & $29(0.0473)$ & $29(0.0522)$ & $29(0.0545)$ & $28(0.0463)$ & $28(0.0478)$ & $22(0.0406)$ \\
\hline & $10^{-1}$ & $27(0.0454)$ & $27(0.0498)$ & $\overline{26(0.0446)}$ & $27(0.0501)$ & $23(0.0423)$ & $21(0.0388)$ \\
\hline & 1 & $26(0.0447)$ & $24(0.0428)$ & $25(0.0432)$ & $21(0.0389)$ & $20(0.0374)$ & $21(0.0404)$ \\
\hline & $10^{2}$ & $31(0.0498)$ & $25(0.0454)$ & $23(0.0405)$ & $18(0.0357)$ & $\overline{19(0.0394)}$ & $21(0.0389)$ \\
\hline & & 0 & $10^{-3}$ & $10^{-2}$ & $10^{-1}$ & 1 & $10^{2}$ \\
\hline \multirow{6}{*}{$32 \times 32$} & $10^{-3}$ & $48(0.3312)$ & $48(0.3218)$ & $47(0.3258)$ & $47(0.3405)$ & $50(0.3308)$ & $26(0.1854)$ \\
\hline & $10^{-2}$ & $44(0.3037)$ & $44(0.2948)$ & $44(0.3038)$ & $47(0.3254)$ & $34(0.2439)$ & $22(0.1586)$ \\
\hline & $10^{-1}$ & $41(0.2866)$ & $41(0.2827)$ & $43(0.2879)$ & $31(0.2077)$ & $23(0.1682)$ & $22(0.1586)$ \\
\hline & 1 & $40(0.2905)$ & $42(0.2807)$ & $28(0.1982)$ & $22(0.1579)$ & $19(0.1393)$ & $22(0.1597)$ \\
\hline & $10^{2}$ & $48(0.3346)$ & $24(0.1729)$ & $21(0.1455)$ & $18(0.1329)$ & $\overline{19(0.1348)}$ & $22(0.1558)$ \\
\hline & & 0 & $10^{-3}$ & $10^{-2}$ & $10^{-1}$ & 1 & $10^{2}$ \\
\hline \multirow{6}{*}{$64 \times 64$} & $10^{-3}$ & $71(3.336)$ & $71(3.311)$ & $76(3.489)$ & $78(3.598)$ & $52(2.386)$ & $24(1.166)$ \\
\hline & $10^{-2}$ & $67(3.063)$ & $\overline{71(3.304)}$ & $72(3.299)$ & $49(2.247)$ & $29(1.368)$ & $20(0.984)$ \\
\hline & $10^{-1}$ & $62(2.854)$ & $66(3.031)$ & $\overline{45(2.047)}$ & $27(1.312)$ & $21(1.127)$ & $20(0.9856)$ \\
\hline & 1 & $63(2.893)$ & $42(1.951)$ & $25(1.178)$ & $20(0.979)$ & $\underline{18(0.906)}$ & $20(0.984)$ \\
\hline & $10^{2}$ & $76(3.692)$ & $23(1.099)$ & $20(0.997)$ & $17(0.854)$ & $\overline{18(0.898)}$ & $20(0.978)$ \\
\hline & & 0 & $10^{-3}$ & $10^{-2}$ & $10^{-1}$ & 1 & $10^{2}$ \\
\hline \multirow{5}{*}{$128 \times 128$} & $10^{-3}$ & $108(28.91)$ & $120(31.55)$ & $121(31.63)$ & $78(20.45)$ & $20(5.64)$ & $8(2.82)$ \\
\hline & $10^{-2}$ & $100(26.08)$ & $\overline{113(29.71)}$ & $74(19.65)$ & $19(5.53)$ & $12(3.73)$ & $7(2.62)$ \\
\hline & $10^{-1}$ & $96(25.04)$ & $66(17.10)$ & $\overline{17(4.93)}$ & $11(3.60)$ & $7(2.57)$ & $7(2.61)$ \\
\hline & 1 & $97(25.31)$ & $16(4.70)$ & $10(3.31)$ & $7(2.62)$ & $6(2.35)$ & $7(2.59)$ \\
\hline & $10^{2}$ & 118(30.97) & $10(3.22)$ & $7(2.62)$ & $6(2.35)$ & $\overline{6(2.38)}$ & $7(2.72)$ \\
\hline
\end{tabular}

matrix $\mathcal{P}_{G V D P S S}^{-1} \mathcal{A}$ has at least $n$ eigenvalues 1 .

\section{Conclusion}

We have presented new convergence properties of the GVDPSS method and the optimal parameters, which minimize the spectral radius of the iteration matrix of the GVDPSS iteration method. The GVDPSS preconditioner, which involves two parameters, covers the relaxed versions of the HSS preconditioner $[11,16]$ when it is applied to solve the symmetric saddle point problems as well as the RDPSS preconditioner [9] and the VDPSS preconditioner [18] when it is applied to solve the nonsymmetric saddle point problems. Some numerical examples have been presented to validate the theoretical analysis and show the effectiveness of the method. Numerical performances have shown that the GVDPSS preconditioner is superior to the RHSS, REHSS and RDPSS preconditioners at accelerating the convergence rates of Krylov subspace iteration methods such as GMRES. The performance of the GVDPSS preconditioner is better than that of the VDPSS preconditioner for small value of the parameter $\beta$, but it is 

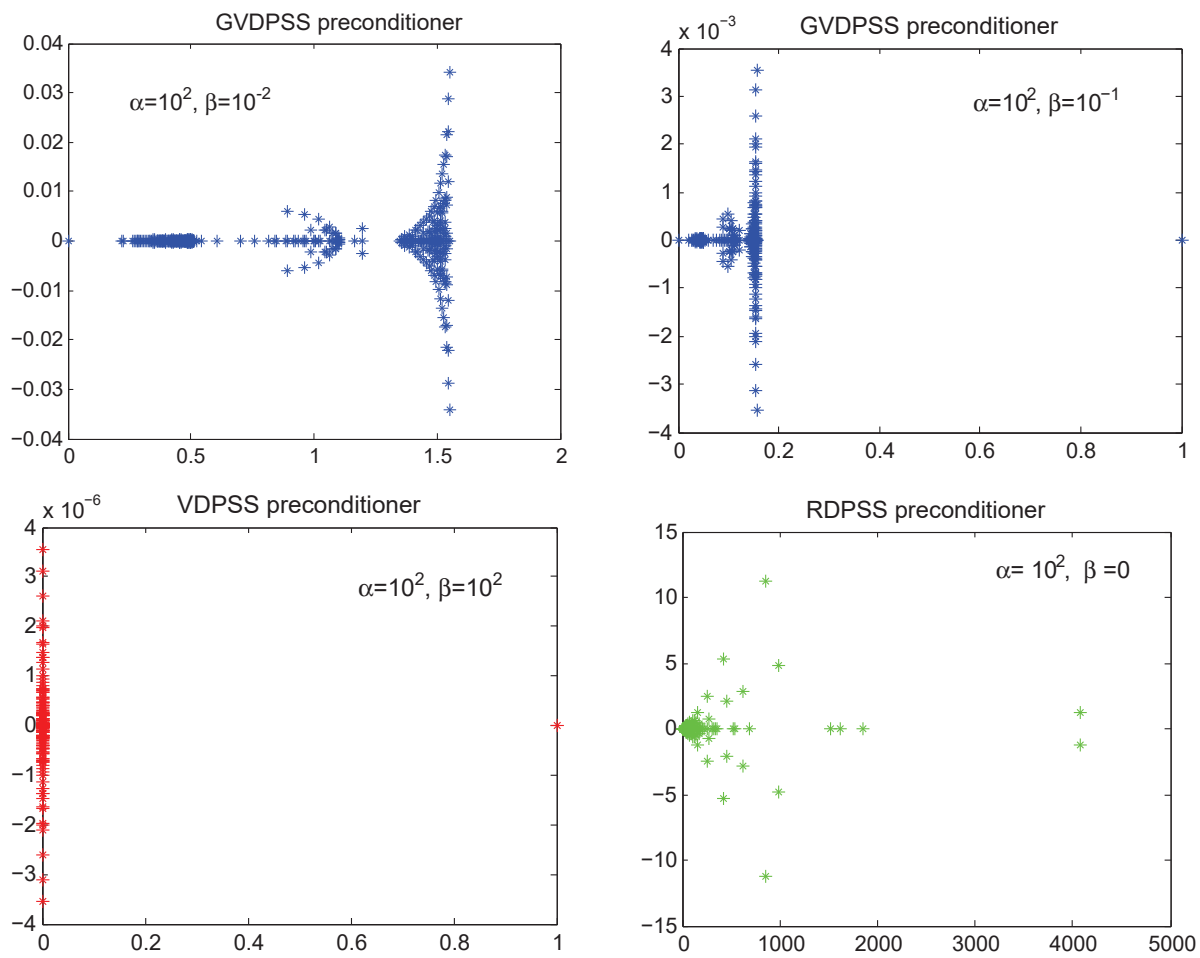

Fig. 3.5. Eigenvalue distribution of the preconditioned matrix for Example $3.2(32 \times 32$ grid).
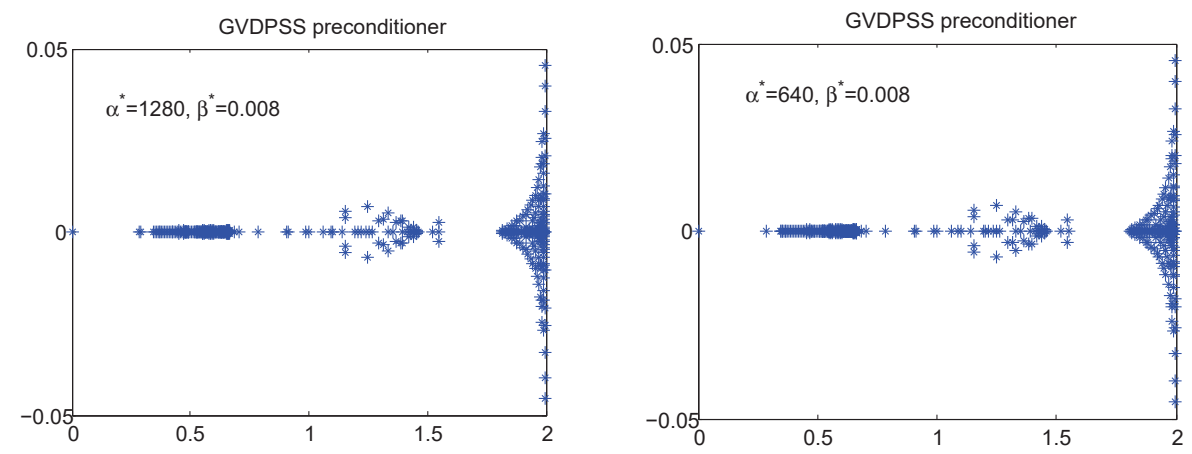

Fig. 3.6. Eigenvalue distribution of the preconditioned matrix with optimal parameters for Example $3.2(32 \times 32$ grid $)$.

not competitive in the case of large $\beta$.

Acknowledgments. The authors are grateful to the anonymous referees and the editor of the journal for their valuable comments and suggestions which improved the quality of this paper. The work of Davod Khojasteh Salkuyeh is partially supported by University of Guilan.

\section{References}

[1] Z.-Z. Bai, Sharp error bounds of some Krylov subspace methods for non-Hermitian linear systems, Appl. Math. Comput., 109 (2000), 273-285. 
[2] Z.-Z. Bai, G.H. Golub, Accelerated Hermitian and skew-Hermitian splitting methods for saddlepoint problems, IMA J. Numer. Anal. 27 (2007), 1-23.

[3] Z.-Z. Bai, G.H. Golub, L.-Z. Lu, J.-F. Yin, Block triangular and skew-Hermitian splitting methods for positive-definite linear systems, SIAM J. Sci. Comput., 26 (2004), 844-863.

[4] Z.-Z. Bai, G.H. Golub, M.K. Ng, Hermitian and skew-Hermitian splitting methods for nonHermitian positive definite linear systems, SIAM J. Matrix Anal. Appl., 24 (2003), 603-626.

[5] Z.-Z. Bai, G.H. Golub, J.-Y. Pan, Preconditioned Hermitian and skew-Hermitian splitting methods for non-Hermitian positive semidefinite linear systems, Numer. Math., 98 (2004), 1-32.

[6] Z.-Z. Bai, J.-F. Yin, Y.-F. Su, A shift-splitting preconditioner for non-Hermitian positive definite matrices, J. Comput. Math., 24 (2006), 539-552.

[7] M. Benzi, G.H. Golub, A preconditioner for generalized saddle point problems, SIAM J. Matrix Anal. Appl., 26 (2004), 20-41.

[8] M. Benzi, G.H. Golub, J. Liesen, Numerical solution of saddle point problems, Acta Numer., 14 (2005), 1-137.

[9] Y. Cao, J.-L. Dong, Y.-M. Wang, A relaxed deteriorated PSS preconditioner for nonsymmetric saddle point problems from the steady Navier-Stokes equation, J. Comput. Appl. Math., 273 (2015), 41-60

[10] Y. Cao, J. Du, Q. Niu, Shift-splitting preconditioners for saddle point problems, J. Comput. Appl. Math., 272 (2014), 239-250.

[11] Y. Cao, L.-Q. Yao, M.-Q. Jiang, Q. Niu, A relaxed HSS preconditioner for saddle point problems from mesh free discretization, J. Comput Math. 31 (2013), 398-421.

[12] H.C. Elman, A. Ramage, D.J. Silvester, IFISS: A Matlab toolbox for modelling incompressible flow, ACM Trans. Math. Software., 33 (2007), Article 14.

[13] Z.-G Huang, L.-G Wang, Z. Xu, J.-J Cui, A generalized variant of the deteriorated PSS preconditioner for nonsymmetric saddle point problems, Numr. Algor. 75 (2017) 1161-1191.

[14] J.-Y. Pan, M.K. Ng, Z.-Z. Bai, New preconditioners for saddle point problems, Appl. Math. Comput., 172 (2006), 762-771.

[15] Y. Saad, Iterative Methods for Sparse Linear Systems, SIAM: Philadelphia, 2003.

[16] D.K. Salkuyeh, M. Masoudi, A new relaxed HSS preconditioner for saddle point problems, Numer. Algor., 74 (2017) 781-795.

[17] E.D. Sturler, J. Liesen, Block-diagonal and constraint preconditioners for nonsymmetric indenite linear systems, SIAM, J. Sci. Comput., 26 (2005), 1598-619.

[18] J. Zhang, C. Gu, A variant of the deteriorated PSS preconditioner for nonsymmetric saddle point problems, BIT Numer. Math., 2016, doi: 10.1007/s10543-015-0590-9. 